\title{
Maximum Quasi-Likelihood Estimation in Fractional Levy Stochastic Volatility Model
}

\author{
Jaya Prakasah Narayan Bishwal \\ Department of Mathematics and Statistics, University of North Carolina at Charlotte, Charlotte, USA \\ E-mail:J.Bishwal@uncc.edu \\ Received July 13, 2011; revised August 12, 2011; accepted August 22, 2011
}

\begin{abstract}
Usually asset price process has jumps and volatility process has long memory. We study maximum quasilikelihood estimators for the parameters of a fractionally integrated exponential GARCH, in short FIECOGARCH process based on discrete observations. We deal with a compound Poisson FIECOGARCH process and study the asymptotic behavior of the maximum quasi-likelihood estimator. We show that the resulting estimators are consistent and asymptotically normal.
\end{abstract}

Keywords: Stochastic Volatility, Long Memory Process, Jump Process, Fractional Levy Process, Poisson Process, Ornstein-Uhlenbeck Process, Gaussian Quasi-Likelihood, Consistency, Asymptotic Normality

\section{Introduction}

Parameter estimation in GARCH models has been studied extensively in view of their wide applications. In order to incorporate long memory in the model we look at the continuous time counterpart. Most of the financial transactions are recorded these days with ultra high frequency which are also called tick-by-tick data. The most important feature of these data are irregular spacing of observation time points. If one aggregates the data up to fixed time intervals, there is loss of information which should be avoided. Hence it is natural to model price processes in continuous time. Also volatility clusters on high level. This long-run volatility persistence is not taken into account by GARCH models. Levy processes contribute non-normality and jumps in the observed part of the model and fractional processes contribute long memory in unobserved part in the model. Based on discrete observations of the price process we estimate all the unknown parameters of the price and the volatility process by the maximum quasi-likelihood estimation. We use Gaussian quasi-likelihood as a contrast function. Maximum quasi-likelihood estimator is an application of Kalman filter in the context of maximum likelihood estimation. Even if the data are non-normal, we use the Gaussian log-likelihood.

Non-negative Ornstein-Uhlenbeck processes were first studied by Wolfe [1] who obtained it as a limit of an autoregressive process with positive innovations. These days non-negative Ornstein-Uhlenbeck processes have received lot of attention in view of their applications as interest rate and stochastic volatility models, see BarndorffNielsen and Shephard [2,3]. Gaver and Lewis [4] studied estimation in first order autoregressive gamma process. Davis and McCormick [5] studied estimation for first order autoregressive process with positive or bounded innovations. Neilsen and Shephard [6] studied likelihood analysis of a first order autoregressive model with exponential innovations. Brockwell, Davis and Yang [7] studied parameter estimation of non-negative Levy driven Ornstein-Uhlenbeck processes. Jongbloed, van der Meulen and van der Vaart [8] studied nonparametric estimation for Levy driven Ornstein-Uhlenbeck processes.

Parameter estimation in Gaussian Ornstein-Uhlenbeck process was extensively studied in Bishwal [9]. To account for the strong persistence in volatility, which sometimes is observed in empirical data, we study the FIECOGARCH (fractionally integrated exponential continuous time generalized autoregressive conditional heteroscedastic) process. The log-price process has jumps and the log-volatility process has long memory. The long memory effect introduced in the log-volatility process, propagates to the volatility process. Thus the model here captures the two stylized facts of long memory and jumps. Continuous time long memory process in volatility received some attention, see Comte and Renault [10] where they used fractional Ornstein-Uhlenbeck process as the stochastic volatility process and Comte, Coutin and Renault 
[11] where they used fractional CIR square root process as the stochastic volatility process. Marquardt [12] studied fractional Levy processes and applied it to long memory moving average processes. Marquardt [13] studied multivariate fractionally integrated CARMA processes. Bender and Marquardt [14] studied stochastic calculus for convoluted Levy processes. Haug and Czado [15] studied structural properties of an exponential continuous time GARCH process. Haug and Czado [16] studied the structural properties of FIECOGARCH model. Czado and Haug [17] used an exponential autoregressive conditional duration model to describe the dependence structure in durations of ultra high frequency financial data. Haug, Kluppelberg, Lindner and Zapp [18] studied method of moment estimation in the $\mathrm{COGARCH}(1,1)$ model. COGARCH model is suitable for irregularly spaced observation times. Straumann and Mikosch [19] studied quasimaximum likelihood estimation in conditionally heteroscedastic time series model. Haug and Czado [20] studied quasi-maximum likelihood estimation and prediction in the compound Poisson ECOGARCH $(1,1)$ model.

\section{Preliminaries}

Fractional Levy Process is a generalization of fractional Brownian motion and is defined as

$M_{H, t}=\frac{1}{\Gamma\left(H+\frac{1}{2}\right)} \int_{R}\left[(t-s)_{+}^{H-1 / 2}-(-s)_{+}^{H-1 / 2}\right] \mathrm{d} M_{s}, t \in \mathbb{R}$

where $H \in(0.5,1), u_{+}=\max (u, 0), u_{-}=\max (-u, 0)$ and $\left\{M_{t}, t \in \mathbb{R}\right\}$ is a Levy process on $\mathbb{R}$ with

$$
E\left(M_{1}\right)=0, E\left(M_{1}^{2}\right)<\infty
$$

and without Brownian component.

Following are the properties of fractional Levy process:

1) The covariance of the FLP is given by

$$
\begin{aligned}
\operatorname{Cov}\left(M_{H, t}, M_{H, s}\right)= & \frac{E\left[L(1)^{2}\right]}{2 \Gamma(2 H+1) \sin (\pi H)} \\
& \times\left[|t|^{2 H}+|s|^{2 H}-|t-s|^{2 H}\right]
\end{aligned}
$$

2) $M_{H}$ is not a martingale. For a large class of Levy processes, $M_{H}$ is neither a semimartingale.

If the Levy process $M$ is of finite activity, then the corresponding fractional Levy process $M_{H}$ is of finite variation. In the case when $M$ is not of finite activity, the corresponding fractional Levy process is not a semimartingale.

3) $M_{H}$ is Hölder continuous of any order $v$ less than $H-\frac{1}{2}$.
4) $M_{H}$ has stationary increments.

5) $M_{H}$ is symmetric.

6) $M_{H}$ is self similar.

7) $M_{H}$ has infinite total variation on compacts.

8) $M_{H}$ is not self similar.

9) $M_{H}$ has long memory.

10) Fractional Levy Ornstein-Uhlenbeck (FLOU). Process:

The FIMA (fractionally integrated moving average) process is defined as

$$
Y_{H}(t)=\int_{-\infty}^{t} g_{H}(t-u) L(\mathrm{~d} u), t \in \mathbb{R}
$$

where $g_{H}(t)=\frac{1}{\Gamma\left(H-\frac{1}{2}\right)} \int_{0}^{t} g(t-s) s^{H-\frac{3}{2}} \mathrm{~d} s, t \in \mathbb{R}$

and the kernel $g$ is the kernel of a short memory moving average process.

The process $Y_{H}(t)$ can be written as

$$
Y_{H}(t)=\int_{-\infty}^{t} g(t-u) \mathrm{d} M_{H, u}, t \in \mathbb{R} .
$$

We assume the following conditions on the kernel $g$ : $\mathbb{R} \rightarrow \mathbb{R}$.

(A1) $g(t)=0$ for all $t<0$ (causality).

(A2) $|g(t)| \leq C e^{-c t}$ for some constants $C>0$ and $C>0$ (short memory).

FIMA process is stationary and is infinite divisible.

Consider the kernel $g(t-s)=\sigma e^{\theta(t-s)} I_{(0, \infty)}(t-s)$ then

$$
g_{H}(t)=\frac{\sigma}{\Gamma\left(H-\frac{1}{2}\right)} \int_{0}^{\infty} e^{\theta(t-s)} I_{(0, \infty)}(t-s) s^{H-\frac{3}{2}} \mathrm{~d} s, t \in \mathbb{R} .
$$

Note that

$$
V_{H, t}^{\theta, \sigma}=\int_{R} g_{H}(t-u) L(\mathrm{~d} u), t \in \mathbb{R}
$$

is the fractional Levy Ornstein-Uhlenbeck (FLOU) process satisfying the fractional Vasicek model

$$
\mathrm{d} V_{H, t}=\theta V_{H, t} \mathrm{~d} t+\sigma \mathrm{d} M_{H, t}, t \in \mathbb{R} .
$$

\section{Main Results}

The FIECOGARCH process is given by $\mathrm{d} Y_{H, t}=\sqrt{X_{H, t}} \mathrm{~d} L_{t}$

$$
\begin{gathered}
X_{H, t}=e^{\mu+\beta V_{H, t_{-}}} \\
\mathrm{d} V_{H, t}=\theta V_{H, t} \mathrm{~d} t+\mathrm{d} M_{H, t}
\end{gathered}
$$

where $L_{t}$ is a Levy process independent of $M_{H, t}$ which is a fractional Levy process of integration (Hurst) parameter $H \in(0.5,0.75)$. 
We estimate $\mu, \beta$ and $\theta$ by the maximum quasilikelihood method and study the asymptotic properties of the estimators.

Equivalently, $\mathrm{d} Y_{H, t}=\mu \mathrm{d} t+\sigma e^{X_{H, t}} \mathrm{~d} L_{t}$

$$
\mathrm{d} X_{H, t}=\theta X_{H, t} \mathrm{~d} t+\mathrm{d} M_{H, t} .
$$

The log-volatility process is strictly stationary and the covariance function is of the form

$$
\operatorname{Cov}\left(\log \left(\sigma_{H, t+h}^{2}\right), \log \left(\sigma_{H, t}^{2}\right) \sim C_{1} h^{2(H-1)} \text { when } h \rightarrow \infty\right. \text {. }
$$

The autocorrelation function of log-volatility and volatility process decay at a hyperbolic rate.

The autocovariance function of the price returns over a time interval of length $r>0$

$$
S_{H, t}^{(r)}=S_{H, t}-S_{H, t-r}=\int_{(t-r, t]} \sigma_{H, s} \mathrm{~d} L_{s}, t \geq r>0
$$

is given by

$$
\operatorname{Cov}\left(\left(S_{H, t}^{(r)}\right)^{2},\left(S_{H, t+h}^{(r)}\right)^{2}\right)=E\left(L_{1}^{2}\right) \int_{h}^{h+r} \operatorname{Cov}\left(\left(S_{H, t}^{(r)}\right)^{2}, \sigma_{s}^{2}\right) \mathrm{d} s .
$$

We will treat the special case of Compound Poisson FIECOGARCH process first. We will estimate the parameters by maximum quasi-likelihood estimation method.

A compound Poisson FIECOGARCH process is driven by the Levy process $L$ with Levy symbol

$$
\psi_{L}(u)=-\frac{u^{2}}{2}+\int_{R}\left(e^{i u x}-1\right) \Phi_{0,1 / \lambda}(\mathrm{d} x),
$$

where $\Phi_{0,1 / \lambda}$ is a normal distribution with mean 0 and variance $1 / \lambda$. This means that $L$ is the sum of a standard Brownian motion $W$ and a compound Poisson process

$$
J_{t}=\sum_{k=1}^{N_{t}} Z_{k}, t \geq 0
$$

where $N_{t}$ is an independent Poisson process with intensity $\lambda>0$ and jump times $\left(T_{k}\right)_{k \in Z}$. The Poisson process is also independent from the i.i.d. sequence of jump sizes $\left(Z_{k}\right)_{k \in Z}$ with $Z_{1} \sim N(0,1 / \lambda)$. The Levy process $M$ in this case is given by

$$
M_{t}=\sum_{k=1}^{N_{t}}\left(\theta Z_{k}+\gamma\left|Z_{k}\right|\right)-C t, t>0
$$

with $\quad C:=\gamma \int_{R}|x| \lambda \Phi_{0,1 / \lambda}(\mathrm{d} x)=\sqrt{\frac{2 \lambda}{\pi}} \gamma$.

The stationary log-volatility is of the form

$$
\log \left(\sigma_{H, t_{+}}^{2}\right)=\mu+\int_{-\infty}^{t} \beta e^{-a_{1}(t-s)} \mathrm{d} M_{H, s}, t>0 .
$$

The log-price process is given by

$$
S_{t}=\sum_{k=1}^{N_{t}} \sigma_{T_{k}} Z_{k}, t \geq 0, S_{0}=0
$$

We observe $S$ at $n$ consecutive jump times

$$
0=T_{0}<T_{1}<\cdots<T_{n}<T<T_{n+1}, n \in \mathbb{Z}
$$

over the time interval $[0, T]$. The state process $X_{H}$ has then the following autoregressive representation

$$
\begin{aligned}
\beta X_{H, T_{i}}= & \beta e^{-\alpha \Delta T_{i}} X_{H, T_{i-1}} \\
& +\sum_{k=N_{T_{i-1}}+1}^{N_{T_{i}}} \beta e^{-\alpha\left(T_{i}-T_{k}\right)}\left[\theta Z_{k}+\gamma\left|Z_{k}\right|\right] \\
& -\int_{T_{i-1}}^{T_{i}} \beta e^{\left(T_{i}-s\right)} C \mathrm{~d} s \\
= & \beta e^{-\alpha \Delta T_{i}} X_{H, T_{i-1}}+\beta \theta Z_{i}+\beta\left(\left|Z_{i}\right|-\frac{C}{\alpha}\left(1-e^{-\alpha \Delta T_{i}}\right)\right)
\end{aligned}
$$

where $\Delta T_{i}:=T_{i}-T_{i-1}, i=1,2, \cdots, n$ and $N_{T_{i-1}}+1=N_{T_{i}}=i$.

Thus $\log \left(\sigma_{H, T_{i}}^{2}\right)=\mu+\beta e^{-\alpha \Delta T_{i}} X_{T_{i-1}}-\beta \frac{C}{\alpha}\left(1-e^{-\alpha \Delta T_{i}}\right)$ since $\beta X_{H, T_{i-}}=\beta X_{H, T_{i-1}}-\beta \frac{C}{\alpha}\left(1-e^{-\alpha \Delta T_{i}}\right)$ as $X_{T_{i-}}$ does not include the jump at time $T_{i}$ and its corresponding jump size is $Z_{i}$.

The leverage effect depends on the sign of $\beta \theta$. We will also identify the leverage parameter $\theta$.

The compound Poisson process has finite activity. The observation of the log-price process is given by

$$
S_{T_{i}}=\sum_{k=1}^{N} \sigma_{T_{i}} Z_{k}=S_{H, T_{i-1}}+\sigma_{H, T_{i}} Z_{i} .
$$

We study the parameter estimation in two steps. The rate $\lambda$ of the Poisson process $N$ can be estimated given the jump times $T_{i}$, therefore it is done at a first step. Since we observe total number of jumps $n$ of the Poisson process $N$ over the $T$ intervals of length one, the MLE of $\lambda$ is given by

$$
\hat{\lambda}_{n}:=\frac{n}{T}
$$

Theorem 3.1 We have

$$
\begin{gathered}
\hat{\lambda}_{n} \rightarrow \lambda \text { a.s. as } n \rightarrow \infty \\
n^{1-H}\left(\hat{\lambda}_{n}-\lambda\right) \rightarrow^{D} N\left(0, e^{\lambda}\left(1-e^{-\lambda}\right)\right) \text { as } n \rightarrow \infty .
\end{gathered}
$$

Proof. Let $U_{i}$ be the number of jumps in the interval $(i-1, i]$. Then $U_{i}, i=1,2, \cdots, n$ are i.i.d. Poisson distributed with parameter $\lambda$. Since $\Phi$ is continuous, we have $I_{\{0\}}\left(U_{i}\right)=I_{\{0\}}\left(S_{H, i}^{(1)}\right)$, a.s. $i=1,2, \cdots, n$. Note that $\frac{1}{n} \sum_{i=1}^{n} I_{\{0\}}\left(S_{H, i}^{(1)}\right) \rightarrow^{a . s .} E\left(I_{\{0\}} U_{1}\right)=P\left(U_{1}=0\right)=e^{-\lambda}$ as $n \rightarrow \infty$ 
LLN and CLT and delta method applied to the sequence $I_{\{0\}}\left(S_{H, i}^{(1)}\right), i=1,2, \cdots, n$ give the results.

The CLT result above allows us to construct confidence interval for the jump rate $\lambda$. A $100(1-\alpha) \%$ confidence interval for $\lambda$ is given by

$$
\left[\frac{n}{T}-q_{1-\frac{\alpha}{2}} \sqrt{\frac{1}{n}-\frac{1}{T}}, \frac{n}{T}+q_{1-\frac{\alpha}{2}} \sqrt{\frac{1}{n}-\frac{1}{T}}\right]
$$

where $q_{1-\frac{\alpha}{2}}$ is the $\left(1-\frac{\alpha}{2}\right)$-quantile of the standard normal distribution.

To estimate the remaining parameters $(\alpha, \theta, \mu)$, we use the quasi maximum likelihood (QML) estimation procedure as developed by Straumann [21] in conditionally heteroscedastic time series models. Since the conditional distributions of the returns is unknown, we will use the QML approach by choosing the Gaussian quasi-likelihood as a contrast function.

The conditional log-likelihood has the representation

$$
\begin{aligned}
L\left(\vartheta \mid S_{H}^{\Delta}, \lambda\right):= & -\frac{n}{2} \log (2 \pi) \\
& -\frac{1}{2}\left(\sum_{i=1}^{n} \log \left(\sigma_{T_{i}}^{2} / \lambda\right)-\sum_{i=1}^{n} \frac{\left(S_{H, T_{i}}^{\Delta T_{i}}\right)^{2}}{\sigma_{T_{i}}^{2} / \lambda}\right) .
\end{aligned}
$$

The quasi log-likelihood function for $\vartheta=(\alpha, \theta, \gamma, \mu)$ given $S_{H}^{\Delta}:=\left(S_{H, T_{1}}^{\Delta T_{1}}, S_{H, T_{2}}^{\Delta T_{2}}, \cdots, S_{H, T_{n}}^{\Delta T_{n}}\right)$ and the MLE $\hat{\lambda}_{n}$ is defined as

$$
\begin{aligned}
L\left(\vartheta||_{H}^{\Delta}, \hat{\lambda}_{n}\right):= & -\frac{1}{2} \sum_{i=1}^{n} \log \left(\hat{\sigma}_{H, T_{i}}^{2}\left(\vartheta, \hat{\lambda}_{n}\right)\right) \\
& -\frac{1}{2} \sum_{i=1}^{n} \frac{\left(S_{H, T_{i}}^{\Delta T_{i}}\right)^{2}}{\hat{\sigma}_{H, T_{i}}^{2}\left(\vartheta, \hat{\lambda}_{n}\right) / \hat{\lambda}_{n}}
\end{aligned}
$$

where the estimates of the volatility $\sigma_{H, T_{i}}^{2}, i=1,2, \cdots, n$ are given by

$$
\begin{aligned}
\hat{\sigma}_{H, T_{i}}^{2}\left(\vartheta, \lambda_{n}\right) & :=\exp \left(\mu+e^{-\alpha \Delta T_{i}} X_{H, T_{i-1}}(\vartheta, \lambda)-\hat{C} \Delta T\right), \\
& =1,2, \cdots, n
\end{aligned}
$$

and given the parameters $\vartheta$ and $\lambda$ the estimates of the state process $X$ are given by the recursion

$$
\begin{aligned}
\hat{X}_{H, T_{i}}= & e^{-\alpha \Delta T_{i}} \hat{X}_{T_{i-1}}+\theta \frac{S_{H, T_{i}}}{\hat{\sigma}_{T_{i}}(\vartheta, \lambda)} \\
& +\left(\frac{S_{H, T_{i}}}{\hat{\sigma}_{T_{i}}(\vartheta, \lambda)}-\hat{C} \Delta T_{i}\right) .
\end{aligned}
$$

Note that $E(|W|)=\sqrt{\frac{2}{\pi \lambda}}, W \sim N(0,1 / \lambda)$.

Here the approximation $\left(1-e^{-z}\right) \approx z$ for small $z$ is used. The recursion needs a starting value $\hat{X}_{H, 0}$ which will be the mean value zero of the stationary distribution of $X$.

MQLE of $\vartheta$ is defined as

$$
\hat{\vartheta}_{n}:=\arg \max _{\vartheta \in \Theta} L\left(\vartheta \mid S^{\Delta}, \hat{\lambda}_{n}\right) .
$$

The parametric estimator of volatility at jump times $0=T_{0}<T_{1}<\cdots<T_{n}<T<T_{n+1}, n \in Z$ is given by

$$
\begin{aligned}
\hat{\sigma}_{H, T_{i}}^{2}\left(\hat{\vartheta}_{n}, \hat{\lambda}_{n}\right) & :=\exp \left(\hat{\mu}_{n}+e^{-\hat{\alpha}_{n} \Delta T_{i}} X_{H, T_{i-1}}\left(\hat{\vartheta}_{n}, \hat{\lambda}_{n}\right)-\hat{C} \Delta T_{i}\right), \\
i & =1,2, \cdots, n
\end{aligned}
$$

which uses the MQLE $\hat{\vartheta}_{n}$.

Let $F_{H}\left(\vartheta_{0}\right)$ be the Fisher information of the log-price process where $\vartheta_{0}$ is the true parameter. The following theorem gives the strong consistency and the asymptotic normality of the MQLE.

Theorem 3.2

a) $\hat{\vartheta}_{n} \rightarrow \vartheta_{0}$ a.s. as $n \rightarrow \infty$

b) $n^{1 / 2}\left(\hat{\vartheta}_{n}-\vartheta_{0}\right) \rightarrow^{D} N\left(0, F_{H}^{-1}\left(\vartheta_{0}\right)\right)$ as $n \rightarrow \infty$

Proof. The discrete time process $S^{\Delta}$ process is strong mixing with geometric rate and since strict stationarity of $\sigma^{2}$ implied strict stationarity of $S^{\Delta}$ is also ergodic. Hence application of the Birkoff ergodic theorem and central limit theorem for strongly mixing process proves the theorem.

Remark. We considered the case $H \in[0.5,0.75)$. For the case $H=0.75$, the asymptotic distribution of the MQLE will be normal with the rate $n(\log n)^{1 / 2}$ and the asymptotic distribution of the MQLE will be nonGaussian Rosenblatt distribution with rate $n^{2 H-1}$. This shows the different behavior of the MQLE in different parts of the Hurst space even in the stationary case. In the nonstationary case, the limit distribution will be mixture normal. Thus not only the original parameter space, but also the Hurst space plays an important role in obtaaining the limit distribution of the MQLE in long memory models.

\section{Conclusions}

We studied parameter estimation in a FIECOGARCH model from discrete data. We obtained the consistency and asymptotic normality of the maximum quasi-likelihood estimator. Here we assumed $H$ to be known. An im- 
portant problem is estimation of $H$, for instance based on second order quadratic variation and remains to be investigated.

\section{References}

[1] A. J. Wolfe, "On a Continuous Analogue of the Stochastic Difference Equation $X_{n}=\rho X_{n}+B_{n}$," Stochastic Processes and their Applications, Vol. 12, No. 3, 1982, pp. 301312. doi:/10.1016/0304-4149(82)90050-3

[2] O. E. Barndorff-Nielsen and N. Shephard, "Non-Gaussian Ornstein-Uhlenbeck-Based Models and Some of Their Uses In Financial Economics (with Discussion)," Journal of the Royal Statistical Society, Series B, Vol. 63, No. 2, 2001, pp. 167-241. doi:/10.1111/1467-9868.00282

[3] O. E. Barndorff-Neilsen and N. Shephard, "Normal Modified Stable Processes," Theory of Probability and Mathematical Statistics, Vol. 65, 2002, pp. 7-20.

[4] D. P. Gaver and P. A. W. Lewis, "First Order Autoregressive Gamma Sequences and Point Processes," Advances in Applied Probability, Vol. 12, No. 3, 1980, pp. 727-745. doi:/10.2307/1426429

[5] R. A. Davis and W. P. McCormick, "Estimation for First Order Autoregressive Processes with Positive or Bounded Innovations," Stochastic Processes and Their Applications, Vol. 31, No. 2, 1989, pp. 237-250. doi:/10.1016/0304-4149(89)90090-2

[6] B. Neilsen and N. Shephard, "Likelihood Analysis of a First Order Autoregressive Model with Exponential Innovations," Journal of Time Series Analysis, Vol. 24, No. 3, 2003, pp. 337-344. doi:/10.1111/1467-9892.00310

[7] P. J. Brockwell, R. A. Davis and Y. Yang, "Estimation for Non-Negative Levy Driven Ornstein-Uhlenbeck Processes," Journal of Applied Probability, Vol. 44, No. 4, 2007, pp. 977-989. doi:/10.1239/jap/1197908818

[8] G. Jongbloed, F. H. van der Meulen and A. W. van der Vaart, "Nonparametric Inference for Levy Driven Ornstein-Uhlenbeck Processes," Bernoulli, Vol. 11, No. 5, 2005, pp. 759-791. doi:/10.3150/bj/1130077593

[9] J. P. N. Bishwal, "Parameter Estimation in Stochastic Differential Equations," Springer-Verlag, Berlin, 2008. doi:/10.1007/978-3-540-74448-1

[10] F. Comte and E. Renault, "Long Memory in Continuous-
Time Stochastic Volatility Models," Mathematical Finance, Vol. 8, No. 4, 1998, pp. 291-323. doi:/10.1111/1467-9965.00057

[11] F. Comte, L. Coutin and E. Renault, "Affine Fractional Stochastic Volatility Models with Application to Option Pricing," Recherche, Vol. 13, No. 1993, 2010, pp. 1-35.

[12] T. Marquardt, "Fractional Levy Processes with ApplicaTion to Long Memory Moving Average Processes," Bernoulli, Vol. 12, No. 6, 2006, pp. 1009-1126. doi:/10.3150/bj/1165269152

[13] T. Marquardt, "Multivariate FICARMA Processes," Journal of Multivariate Analysis, Vol. 98, No. 9, 2007, pp. 1705-1725. doi:/10.1016/j.jmva.2006.07.001

[14] C. Bender and T. Marquardt, "Stochastic Calculus for Convoluted Levy Processes," Bernoulli, Vol. 14, No. 2, 2007, pp. 499-518. doi:/10.3150/07-BEJ115

[15] S. Haug and C. Czado, "An Exponential Continuous Time GARCH Process," Journal of Applied Probability, Vol. 44, No. 4, 2007, pp. 960-976. doi:/10.1239/jap/1197908817

[16] S. Haug and C. Czado, "Fractionally Integrated ECOGARCH Process," Sonderforschungsbereich, Vol. 386, No. 484, 2006.

[17] C. Czado and S. Haug, "An ACD-ECOGARCH $(1,1)$ Model," Journal of Financial Econometrics, Vol. 8, No. 3, 2010, pp. 335-344. doi:/10.1093/jifinec/nbp023

[18] S. Haug, C. Kluppelberg, A. Lindner and M. Zapp, "Method of Moment Estimation in the COGARCH(1,1) Model," Econometrics Journal, Vol. 10, No. 2, 2007, pp. 320-341. doi:/10.1111/j.1368-423X.2007.00210.x

[19] D. Straumann and T. Mikosch, "Quasi-Maximum Likelihood Estimation in Conditionally Heteroscedastic Time Series: A Stochastic Recurrence Equations Approach," Annals of Statistics, Vol. 34, 2006, pp. 2449-2495. doi:/10.1214/009053606000000803

[20] S. Haug and C. Czado, "Quasi Maximum Likelihood Estimation and Prediction in the Compound Poisson ECOGARCH $(1,1)$ Model," Sonderforschungsbereich, Vol. 386, No. 516, 2006.

[21] D. Straumann, "Estimation in Conditionally Heteroscedastic Time Series Models," Lecture Notes in Statistics, Vol. 181, Springer-Verlag, Berlin, 2005. 